\author{
Wenjing Sun \\ Hongquan Lu \\ Shujuan Dong \\ Rui Li \\ Yingjie Chu \\ Wang Nan \\ Yu Zhao \\ Yabin Zhang \\ Limeiting Wang \\ Lin Sun \\ Di Lu
}

5 Research Square

Preprints are preliminary reports that have not undergone peer review.

They should not be considered conclusive, used to inform clinical practice, or referenced by the media as validated information.

\section{Beclin- 1 controls caspase-4 inflammasome activation and pyroptosis after myocardial injury}

\section{Video Byte}

Keywords: Beclin-1, BECN1, caspase-4 inflammasome, pyroptosis, reperfusion, ischemia, ischemia/reperfusion, I/R, microvascular injury, cardiovascular disease, autophagy, infarct, cardiology, myocardial infarction, AMI, microvascular obstruction, no-reflow, gasdermin D, GSDMD, Cell Communication and Signaling

Posted Date: March 1st, 2022

DOI: https://doi.org/10.21203/rs.3.rs-1405913/v1

License: (c) (i) This work is licensed under a Creative Commons Attribution 4.0 International License. Read Full License 


\section{Abstract}

Reperfusion of heart tissue with blood after interruption of the blood supply (ischemia) often contributes to inflammation and cell death, including a specific form of cell death called pyroptosis, which can be regulated by the protein caspase- 4 . The protein beclin- 1 is involved in an intracellular degradation process called autophagy that can limit cell death. However, whether beclin-1 limits caspase-4-mediated pyroptosis after heart reperfusion injury remains unclear. To find out, a recent study examined the effects of beclin-1 overexpression in mouse hearts and cultured human heart cells subjected to ischemia/reperfusion. Ischemia/reperfusion increased caspase-4 activity and the expression of the pyroptosis protein gasdermin D. In contrast, beclin-1 overexpression decreased caspase-4 activity, gasdermin D expression, and the levels of the inflammation molecule IL-1 $\beta$. Beclin-1 overexpression also promoted autophagy in the mouse hearts and human cells, and it increased mouse survival and reduced the extent of tissue damage after ischemia/reperfusion. Although the roles of other cell death pathways need to be clarified, the results show that beclin-1-driven autophagy suppresses caspase-4-mediated pyroptosis, which may help protect small blood vessels from injury after ischemia/reperfusion and that inducing beclin-1 signaling may help limit heart reperfusion-induced injury. 